\title{
OLDER ADULTS HOSPITALIZED WITH COVID-19: CLINICAL CHARACTERISTICS AND EARLY OUTCOMES FROM A SINGLE CENTER IN ISTANBUL, TURKEY
}

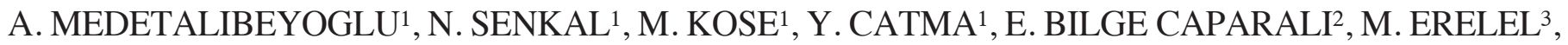 \\ M. ORAL ONCUL ${ }^{4}$, G. BAHAT ${ }^{5}$, T. TUKEK ${ }^{1}$
}

1. Istanbul University Istanbul Faculty of Medicine, Department of Internal Medicine, Istanbul, Turkey; 2. Istanbul University Istanbul Faculty of Medicine, Istanbul, Turkey; 3. Istanbul University Istanbul Faculty of Medicine, Department of Chest Disease, Istanbul, Turkey; 4. Istanbul University Istanbul Faculty of Medicine, Department of Infectious Diseases, Istanbul, Turkey; 5. Istanbul University Istanbul Faculty of Medicine, Department of Internal Medicine, Division of Geriatrics, Istanbul, Turkey. Corresponding author: Gulistan Bahat, Istanbul Universitesi Istanbul Tip Fakultesi, Istanbul, Turkey, gbahatozturk@yahoo.com

\begin{abstract}
Objective: Older adults have been continuously reported to be at higher risk for adverse outcomes of Covid-19. We aimed to describe clinical characteristics and early outcomes of the older Covid-19 patients hospitalized in our center comparatively with the younger patients, and also to analyze the triage factors that were related to the in-hospital mortality of older adults. Design: Retrospective; observational study. Setting: Istanbul Faculty of Medicine hospital, Turkey. Participants: 362 hospitalized patients with laboratoryconfirmed Covid-19 from March 11 to May 11, 2020. Measurements: The demographic information; associated comorbidities; presenting clinical, laboratory, radiological characteristics on admission and outcomes from the electronic medical records were analyzed comparatively between the younger $(<65$ years) and older $(\geq 65$ years) adults. Factors associated with in-hospital mortality of the older adults were analyzed by multivariate regression analyses. Results: The median age was 56 years (interquartile range [IQR], 46-67), and 224 (61.9\%) were male. There were $104(28.7 \%)$ patients $\geq 65$ years of age. More than half of the patients $(58 \%)$ had one or more chronic comorbidity. The three most common presenting symptoms in the older patients were fatigue/ myalgia $(89.4 \%)$, dry cough $(72.1 \%)$, and fever $(63.5 \%)$. Cough and fever were significantly less prevalent in older adults compared to younger patients ( $\mathrm{p}=0.001$ and 0.008 , respectively). Clinically severe pneumonia was present in $31.5 \%$ of the study population being more common in older adults $(49 \%$ vs. $24.4 \%)(\mathrm{p}<0.001)$. The laboratory parameters that were significantly different between the older and younger adults were as follows: the older patients had significantly higher CRP, D-dimer, TnT, pro-BNP, procalcitonin levels, higher prevalence of lymphopenia, neutrophilia, increased creatinine, and lower hemoglobin, ALT, albumin level $(\mathrm{p}<0.05)$. In the radiological evaluation, more than half of the patients (54.6\%) had moderate-severe pneumonia, which was more prevalent in older patients $(66 \%$ vs. $50 \%)(\mathrm{p}=0.006)$. The adverse outcomes were significantly more prevalent in older adults compared to the younger patients (ICU admission, $28.8 \%$ vs. $8.9 \%$; mortality, $23.1 \%$ vs. $4.3 \%$, $\mathrm{p}<0.001)$. Among the triage evaluation parameters, the only factor associated with higher mortality was the presence of clinically severe pneumonia on admission (Odds Ratio $=12.3,95 \%$ confidence interval $=2.7-55.5$, $\mathrm{p}=0.001)$. Conclusion: Older patients presented with more prevalent chronic comorbidities, less prevalent symptomatology but more severe respiratory signs and laboratory abnormalities than the younger patients. Among the triage assessment factors, the clinical evaluation of pulmonary involvement came in front to help clinicians to stratify the patients for mortality risk.
\end{abstract}

Key words: Covid-19, elderly, clinical, characteristics, mortality.

\section{Introduction}

It was early December 2019 that atypical cases of pneumonia were described due to a previously unknown pathogen in Wuhan city, China (1). The pathogen was identified as a novel, positive-sense single-stranded, enveloped RNA virus that has a close similarity with SARS-CoV and named SARS-CoV-2 (2). The associated disease was then named as Coronavirus disease 2019 (Covid-19). It rapidly spread through the World, becoming a pandemic and global health emergency, which affected over 10 million people with a death toll of more than 500 thousand by July 2020 (3). While the disease has been relatively controlled in some regions, when considered globally, it is still in the acceleration phase (3). It is a new disease, and our clinical understanding of the disease

relies on the studies that report its clinical course and associated factors. The early reports in this regard came from China, where the disease started from and continued with Europe and the US in line with its spread pattern. Older adults have been continuously reported to be at higher risk for adverse outcomes of Covid-19 (4-6). However, the specific information about older patients is limited despite their significant involvement with the disease (6-12).

Our hospital is a major teaching hospital. We identified the first Covid-19 case in Turkey in Istanbul city on March 11, 2020 , in the emergency clinic of our university hospital. Since then, our center has become a pandemic hospital followed by the many others in Turkey. Istanbul has been the major center of the pandemic in Turkey with the highest number of Covid19 cases from the beginning of the pandemic in the country 


\section{THE JOURNAL OF NUTRITION, HEALTH \& AGING}

as it is a crossroad between Europe and Asia, an international hub. Istanbul is also the most crowded city in the country, with a population number of about 15 million, higher than many European countries (13). Istanbul has been reported to have more than half $(54.8 \%)$ of all of the cases in Turkey as of June 28, 2020 (14). There are differences between countries in terms of, e.g. demographics, comorbidities, and also in actions taken during the pandemic. These may reflect as differences in presenting clinical features and outcomes (5). Our center has been one of the most crowded centers in the pandemic era in Turkey. In this report, we aimed to describe clinical characteristics and early outcomes of the older Covid-19 patients consecutively hospitalized in our center comparatively with the younger patients. A secondary aim was to analyze the triage factors that were related to the in-hospital mortality of older adults.

\section{Material and Methods}

In this retrospective observational study, we included the hospitalized patients aged $\geq 18$ years with confirmed Covid19 from March 11, 2020 to May 11, 2020. All patients were followed until meeting the outcome measure, death, or discharge from the hospital with recovery.

We recorded demographic information, associated comorbidities presenting clinical, laboratory, and radiological characteristics on admission from the electronic medical records. In our center, after the World Health Organization (WHO)'s declaration of Covid-19 as a public emergency with international concern on January 30, 2020, all of the patients with respiratory symptoms and/or fever were directed to the emergency clinic. All patients were first evaluated in the triage room, where they were assessed in a structured manner involving Covid-19 directed history taking and measurements of the body temperature, pulse rate, respiration rate, blood pressure, and room air peripheral oxygen saturation (SaO2). The history included inquiries on the Covid-19 classical symptoms (i.e., fever, cough and dyspnea), myalgia/ fatigue, contact history, and international travel history. After observation and reports that Covid-19 patients occasionally complained from diarrhea, nausea/vomiting, loss of smell and taste symptoms, these symptoms were also considered as potential symptoms of the disease and included in the structured history on March 25, 2020. The patients that were identified as suspected Covid-19 cases have undergone nasopharyngeal and oropharyngeal swab specimen collection for real-time reverse-transcriptase-polymerase-chain-reaction (RT-PCR) examination, routine laboratory examination, and chest X-ray. Laboratory confirmation (RT-PCR examination) of SARS-CoV-2 was performed at the official public health care laboratory till March 30, 2020, and consequently at our institution certified in this process. RT-PCR assays were carried out in accordance with the protocol defined by the WHO (15). Laboratory examination included complete blood count, blood chemistry (liver and renal function tests, LDH, albumin), CRP, ferritin, D-dimer, high sensitive troponin T (TnT), N-terminal pro-brain natriuretic peptide (pro-BNP) and procalcitonin. In all hospitalized patients with confirmed Covid-19, low dose pulmonary computerized tomography (CT) was also performed at baseline if there were no contraindication (i.e., pregnancy). Pulmonary CT images were evaluated by a structured manner by the attending radiologists that have taken care of the Covid19 pandemic patients. Covid-19 related CT findings were classified as mild to moderate or severe, as described elsewhere (16). Additionally, we evaluated the clinical severity of Covid19 pneumonia on admission, considering the respiration rate, peripheral oxygen saturation, and dyspnea identified by the attending physician. The patients that had resting respiration rate $\geq 30 /$ min or room air peripheral oxygen saturation $<90 \%$ or dyspnea (identified by the use of accessory respiration muscles at rest) were designated as clinically severe pneumonia according to the diagnostic and treatment guidelines for SARS-CoV-2 issued by the Turkish National Scientific Committee (17). The need for oxygen support was assessed by the presence of resting oxygen saturation in the room air $<93 \%$ (17). Body temperature was assessed by a noncontact infrared thermometer from the forehand. Fever was defined as temperature $>38.3^{\circ} \mathrm{C}$. The temperatures between $37.8-38.3$ ${ }^{\circ} \mathrm{C}$ were defined as subfebrile. The cut-offs of the laboratory parameters were designated by the local laboratory thresholds.

Hospitalization criteria: Hospitalization criteria were defined by the national scientific board Covid-19 diagnosis and treatment guideline-directed by the ministry of health (17). Accordingly, the patients that had respiration rate $\geq 30$ / min, room air $\mathrm{SaO} 2<90 \%$; moderate or severe pneumonia on pulmonary imaging; and/or laboratory results associated with a worse prognosis on admission (lymphocyte $<0.8$ x $10^{9} / \mathrm{L}$, CRP $>40 \mathrm{mg} / \mathrm{L}$, ferritin $>500 \mathrm{ng} / \mathrm{mL}$ or D-dimer $>1000 \mathrm{ug} / \mathrm{L})$, and/or the patients that had mild pneumonia but associated chronic comorbidities (e.g. cardiovascular diseases, diabetes mellitus, hypertension, cancer, chronic lung diseases, immunosuppression etc.) and/or older age ( $\geq 50$ years) have been hospitalized (17). The treatment schedule of the hospitalized patients was decided by a constant team and composed of mainly supportive treatment. The additional treatments included hydroxychloroquine, azithromycin, \pm oseltamivir, anticoagulant/antiaggregant treatments, antiviral treatments (lopinavir/ritonavir, favipiravir), anti-inflammatory treatments (steroid, tocilizumab, anakinra), antibiotics largely in concordance with the recommended treatment schedule of the national scientific board.

Admission to the intensive care unit (ICU) and the outcome measure: discharge from hospital or death were recorded from the electronic medical records. We recorded the times between the potential earliest date of symptom onset to hospitalization, hospitalization to ICU admission, length of ICU stay, and length of hospital stay, as applicable.

The study was approved by the institutional review board 
OLDER ADULTS HOSPITALIZED WITH COVID-19

Table 1

Baseline demographic and clinical characteristics of the hospitalized Covid-19 patients stratified by age

\begin{tabular}{|c|c|c|c|c|c|}
\hline Characteristic & & $\begin{array}{l}\text { All Patients }(n=362) \\
(100 \%)\end{array}$ & $\begin{array}{c}<65 \text { y }(n=258) \\
(71.3 \%)\end{array}$ & $\begin{array}{c}\geq 65 \text { y }(n=104) \\
(28.7 \%)\end{array}$ & p \\
\hline Age & & $56(46-67)$ & $50(42-57)$ & $73(68-81)$ & $<0.001 *$ \\
\hline \multirow[t]{3}{*}{ Sex } & & & & & 0.74 \\
\hline & Male & $224(61.9 \%)$ & $161(62.4 \%)$ & $63(60.6 \%)$ & \\
\hline & Female & $138(38.1 \%)$ & $97(37.6 \%)$ & $41(39.4 \%)$ & \\
\hline International travel history & & $6(1.7 \%)$ & $5(1.9 \%)$ & $1(1 \%)$ & 0.67 \\
\hline Contact history & & $134(37 \%)$ & $104(40.3 \%)$ & $30(28.8 \%)$ & $0.04 *$ \\
\hline Smoker & & $36(9.9 \%)$ & $34(13.2 \%)$ & $2(1.9 \%)$ & $0.001 *$ \\
\hline Alcohol & & $2(0.6 \%)$ & none & $2(1.9 \%)$ & 0.08 \\
\hline \multicolumn{6}{|l|}{ Co-morbidities } \\
\hline & Any & $210(58 \%)$ & $126(48.8 \%)$ & $84(80.8 \%)$ & $<0.001 *$ \\
\hline & Hypertension & $138(38.1 \%)$ & $73(28.3 \%)$ & $65(62.5 \%)$ & $<0.001 *$ \\
\hline & COPD/asthma & $41(11.3 \%)$ & $25(9.7 \%)$ & $16(15.4 \%)$ & 0.12 \\
\hline & Coronary heart disease & $37(10.2 \%)$ & $11(4.3 \%)$ & $26(25 \%)$ & $<0.001 *$ \\
\hline & Congestive heart failure & $19(5.2 \%)$ & $3(1.2 \%)$ & $16(15.4 \%)$ & $<0.001 *$ \\
\hline & Cerebrovascular disease & $7(1.9 \%)$ & $3(1.2 \%)$ & $4(3.8 \%)$ & 0.10 \\
\hline & Chronic renal failure & $8(2.2 \%)$ & $5(1.9 \%)$ & $3(2.9 \%)$ & 0.69 \\
\hline & Liver disease & $4(1.1 \%)$ & $1(0.4 \%)$ & $3(2.9 \%)$ & 0.07 \\
\hline & Malignancy & $27(7.5 \%)$ & $12(4.7 \%)$ & $15(14.4 \%)$ & $0.001 *$ \\
\hline & Immunosupression & $18(5 \%)$ & $13(5 \%)$ & $5(4.8 \%)$ & 0.92 \\
\hline \multirow[t]{10}{*}{ Number of co-morbidities Symptoms } & & $1(0-2)$ & $0(0-1)$ & $2(1-2.7)$ & $<0.001 *$ \\
\hline & Fever & $265(73.2 \%)$ & $199(77.1 \%)$ & $66(63.5 \%)$ & $0.008^{*}$ \\
\hline & Cough & $304(84 \%)$ & $227(88 \%)$ & $77(74 \%)$ & $0.001 *$ \\
\hline & Dry cough & $294(81.2 \%)$ & $219(84.9 \%)$ & $75(72.1 \%)$ & $0.005^{*}$ \\
\hline & Dyspnea & $158(43.6 \%)$ & $106(41.1 \%)$ & $52(50 \%)$ & 0.12 \\
\hline & Fatigue/myalgia & $328(90.6 \%)$ & $235(91.1 \%)$ & $93(89.4 \%)$ & 0.62 \\
\hline & Sputum production & $10(2.8 \%)$ & $8(3.1 \%)$ & $2(1.9 \%)$ & 0.73 \\
\hline & Nausea $(n=340)$ & $59(17.4 \%)$ & $47(18.2 \%)$ & $12(12.9 \%)$ & 0.18 \\
\hline & Diarrhea $(n=340)$ & $47(13.8 \%)$ & $36(14 \%)$ & $11(11.8 \%)$ & 0.51 \\
\hline & Loss of smell/taste $(\mathrm{n}=340)$ & $26(7.6 \%)$ & $23(9.3 \%)$ & $3(3.2 \%)$ & 0.068 \\
\hline \multicolumn{6}{|l|}{ Signs } \\
\hline & Body temperature $\left({ }^{\circ} \mathrm{C}\right)$ & $37.2(36.5-38.2)$ & $37.2(36.5-38.2)$ & $37.3(36.5-38.2)$ & 0.80 \\
\hline & Fever $\left(>38.3^{\circ} \mathrm{C}\right)$ & $71(19.6 \%)$ & $53(20.5 \%)$ & $18(17.3 \%)$ & 0.48 \\
\hline & Subfebrile temperature $\left(37.8-38.3^{\circ} \mathrm{C}\right)$ & $49(13.5 \%)$ & $28(10.9 \%)$ & $21(20.2 \%)$ & $0.02 *$ \\
\hline & Dyspnea (use of accessory muscles) & $113(31.2 \%)$ & $62(24 \%)$ & $51(49 \%)$ & $<0.001 *$ \\
\hline & Respiration rate (/min) & $18(16-23)$ & $18(16-22)$ & $20(18-26)$ & $<0.001 *$ \\
\hline \multicolumn{6}{|l|}{ Respiration rate } \\
\hline & $\geq 30 / \min$ & $25(6.9 \%)$ & $11(4.3 \%)$ & $14(13.5 \%)$ & $0.002 *$ \\
\hline & $\mathrm{SaO} 2(\%)$ & $96(93-97)$ & $96(94-98)$ & $94(89-96)$ & $<0.001 *$ \\
\hline & $\mathrm{SaO} 2<90 \%$ & $13(13 \%)$ & $21(8.1 \%)$ & $26(25 \%)$ & $<0.001 *$ \\
\hline & Pulse rate (/min) & $94(86-105)$ & $94(88-105)$ & $95(84.2-102)$ & 0.30 \\
\hline \multicolumn{2}{|l|}{ Need for oxygen support $(\mathrm{SaO} 2<93 \%)$} & $74(20.4 \%)$ & $38(14.7 \%)$ & $36(34.6 \%)$ & $<0.001 *$ \\
\hline \multicolumn{2}{|l|}{ Clinically severe pneumonia } & $114(31.5 \%)$ & $63(24.4 \%)$ & $51(49 \%)$ & $<0.001 *$ \\
\hline \multicolumn{2}{|c|}{ Time from first symptom to hospitalization (d) } & $5(3-7)$ & $5(3-7)$ & $5(3-7)$ & 0.42 \\
\hline
\end{tabular}


THE JOURNAL OF NUTRITION, HEALTH \& AGING

Table 1

General characteristics of the subjects by sex and age

\begin{tabular}{|c|c|c|c|c|}
\hline Characteristics & Full sample $(n=4125)$ & Men $(n=1748)$ & Women $(n=2377)$ & P value for sex \\
\hline Mean age (SD) & $69.2(6.8)$ & $69.7(7.0)$ & $68.8(6.6)$ & 0.001 \\
\hline Mean height m (SD) & $1.56(0.09)$ & $1.63(0.08)$ & $1.51(0.07)$ & 0.001 \\
\hline Mean body mass kg (SD) & $65.3(13.0)$ & $68.3(12.4)$ & $63.3(13.0)$ & 0.001 \\
\hline Mean BMI kg/m2 (SD) & $27.5(5.0)$ & $26.2(4.0)$ & $28.4(5.4)$ & 0.001 \\
\hline \multicolumn{5}{|l|}{ Socioeconomic status, \% } \\
\hline Level I-II (low) & 75.1 & 78.2 & 73.0 & 0.001 \\
\hline \multicolumn{5}{|l|}{ Urbanicity, \% } \\
\hline Urban & 78.4 & 74.3 & 81.3 & 0.001 \\
\hline Rural & 21.6 & 25.7 & 18.7 & \\
\hline \multicolumn{5}{|l|}{ Ethnic group, \% } \\
\hline Indigenous & 6.9 & 9.1 & 5.4 & 0.010 \\
\hline \multicolumn{5}{|l|}{ Frailty health status, $\%$} \\
\hline \multicolumn{5}{|l|}{ Fried criteria } \\
\hline Nonfrail & 8.1 & 11.6 & 5.4 & 0.001 \\
\hline Pre-frail & 63.7 & 67.8 & 60.6 & \\
\hline Frail & 28.2 & 20.6 & 34.0 & \\
\hline \multicolumn{5}{|l|}{ FRAIL scale* } \\
\hline Nonfrail & 41.2 & 48.1 & 36.3 & 0.001 \\
\hline Pre-frail & 54.4 & 49.7 & 57.7 & \\
\hline Frail, \% & 4.4 & 2.2 & 6.0 & \\
\hline Specific subjective memory complaints, $\%$ & 12.2 & 13.2 & 12.2 & 0.318 \\
\hline Hypertension & 53.3 & 45.7 & 59.0 & 0.001 \\
\hline Cardiovascular diseases & 15.8 & 13.6 & 17.5 & 0.001 \\
\hline Stroke & 1.5 & 1.6 & 1.4 & 0.335 \\
\hline Diabetes & 15.8 & 13.6 & 17.5 & 0.001 \\
\hline Cancer & 4.6 & 3.9 & 5.1 & 0.076 \\
\hline Respiratory diseases & 9.9 & 8.6 & 10.8 & 0.009 \\
\hline Osteo-arthrosis & 26.2 & 15.3 & 34.3 & 0.001 \\
\hline Osteoporosis & 10.9 & 4.0 & 16.1 & 0.001 \\
\hline \multicolumn{5}{|l|}{ Physical performance } \\
\hline Mean SPPB score (SD) & $8.8(1.9)$ & $9.3(1.9)$ & $8.5(1.9)$ & 0.001 \\
\hline Mean gait speed, m/s (SD) & $0.76(0.25)$ & $0.81(0.26)$ & $0.72(0.23)$ & 0.001 \\
\hline Mean grip strength, $\mathrm{kg}(\mathrm{SD})$ & $21.1(8.1)$ & $27.0(7.7)$ & $17.0(5.2)$ & 0.001 \\
\hline Low grip strength, $\%$ & 70.8 & 63.5 & 76.2 & 0.001 \\
\hline Mean five times sit to stand test, $\mathrm{s}$ (SD) & $13.7(6.5)$ & $13.2(6.4)$ & $14.1(6.5)$ & 0.001 \\
\hline
\end{tabular}

Data are presented as mean (SD) or percentage of participants. SD, standard deviation; BMI, Body mass index. * Others (mestizo, gitano/ROM, etc). *n=3771 


\section{OLDER ADULTS HOSPITALIZED WITH COVID-19}

Table 2

Parameters of the ROC analysis for the diagnostic performance of SPPB in identifying high risk for frailty phenotype and geriatric syndromes for community-dwelling older adults by sex

\begin{tabular}{|c|c|c|c|}
\hline & Parameter & Frailty by Fried et al. & Frailty by FRAIL Scale \\
\hline \multirow[t]{11}{*}{ Men } & AUC (SE) & $0.742(0.0156)$ & $0.744(0.0572)$ \\
\hline & $95 \% \mathrm{CI}$ & $0.719-0.763$ & $0.722-0.766$ \\
\hline & P-value & $<0.0001$ & $<0.0001$ \\
\hline & Youden index & 0.3828 & 0.3572 \\
\hline & Cut-off & $\leq 8$ & $\leq 8$ \\
\hline & Sensitivity (95\% CI) & $60.24(54.7-65.6)$ & $65.00(48.8-84.6)$ \\
\hline & Specificity $(95 \%$ CI) & $78.03(75.6-80.3)$ & $70.72(68.4-73.0)$ \\
\hline & $+\mathrm{LR}(95 \% \mathrm{CI})$ & $2.74(2.4-3.1)$ & $2.22(1.6-3.1)$ \\
\hline & $-\mathrm{LR}(95 \% \mathrm{CI})$ & $0.51(0.5-0.6)$ & $0.49(0.3-0.9)$ \\
\hline & $+\mathrm{PV}(95 \% \mathrm{CI})$ & $41.6(37.1-46.1)$ & $2.7(1.5-4.7)$ \\
\hline & $-\mathrm{PV}(95 \% \mathrm{CI})$ & $88.1(86.3-90.2)$ & $99.4(98.7-99.7)$ \\
\hline \multirow[t]{11}{*}{ Women } & AUC (SE) & $0.690(0.0122)$ & $0.703(0.0272)$ \\
\hline & $95 \% \mathrm{CI}$ & 0.670 to 0.710 & 0.683 to 0.723 \\
\hline & $P$ value & $<0.0001$ & $<0.0001$ \\
\hline & Youden index & 0.2819 & 0.3067 \\
\hline & Cut-off & $\leq 7$ & $\leq 7$ \\
\hline & Sensitivity (95\% CI) & $46.67(42.9-50.4)$ & $57.14(47.1-68.8)$ \\
\hline & Specificity $(95 \%$ CI) & $81.52(79.4-83.5)$ & $73.52(71.5-75.5)$ \\
\hline & $+\mathrm{LR}(95 \% \mathrm{CI})$ & $2.53(2.2-2.9)$ & $2.16(1.8-2.6)$ \\
\hline & $-\mathrm{LR}(95 \% \mathrm{CI})$ & $0.65(0.6-0.7)$ & $0.58(0.5-0.7)$ \\
\hline & $+\mathrm{PV}(95 \% \mathrm{CI})$ & $56.5(52.4-60.6)$ & $10.3(7.9-13.0)$ \\
\hline & $-\mathrm{PV}(95 \% \mathrm{CI})$ & $74.8(72.5-77.0)$ & $97.0(96.0-97.8)$ \\
\hline
\end{tabular}

AUC: area under the curve; SE: standard error; CI: confidence interval; LR (+): positive likelihood ratio; LR (-): negative likelihood ratio.

(number: 2020/747). The data were collected for routine clinical practice and handled anonymously.

\section{Statistical Analysis}

We expressed continuous variables as medians and interquartile ranges. Categorical variables were given as counts and percentages. Two groups were compared with Mann Whitney U test when necessary. Chi-square test with Yates correction and Fisher's exact test was used for 2X2 contingency tables when appropriate for non-numerical data. Correlations between numerical parameters were analyzed with Spearman's rho correlation test. We made univariate and multivariate regression analyses (Cox survival and logistic regression, backward LR method) to analyze factors associated with mortality in older adults. In the regression analyses, we included the variables that were significantly associated with mortality in the univariate analyses and were clinically significant. The multicollinearity among the possible regression analyses independent variables were checked with Spearman or Kendall's tau-b correlation analyses. The variables that were detected having multicollinearity in between were not analyzed in the same regression models. In the regression analyses, as there were a high number of variables associated with mortality in the univariate analyses, two events per predictor variable were used in regression analyses (18). p values less than 0.05 were accepted as significant. The significance of the regression analyses was checked, and the significance of the logistic regression was further evaluated by Hosmer and Lemeshow test. We used SPSS (statistical package for social sciences) for Windows 15.0 program for data analyses.

\section{Results}

\section{Baseline demographics and clinical characteristics}

We outlined the baseline demographic and clinical characteristics of the patients stratified by age in Table 1 . There were 362 patients with laboratory-confirmed Covid-19 hospitalized in the study period. The date at which the outcome measure (death or discharge from hospital) was completed was June 02,2020 . The median age was 56 years (interquartile range [IQR], 46-67; range, 21-90), and 224 (61.9\%) were male. There were $104(28.7 \%)$ patients $\geq 65$ years of age. The 
THE JOURNAL OF NUTRITION, HEALTH \& AGING

Table 2

Laboratory and radiological findings of the hospitalized Covid-19 patients at hospital admission stratified by age

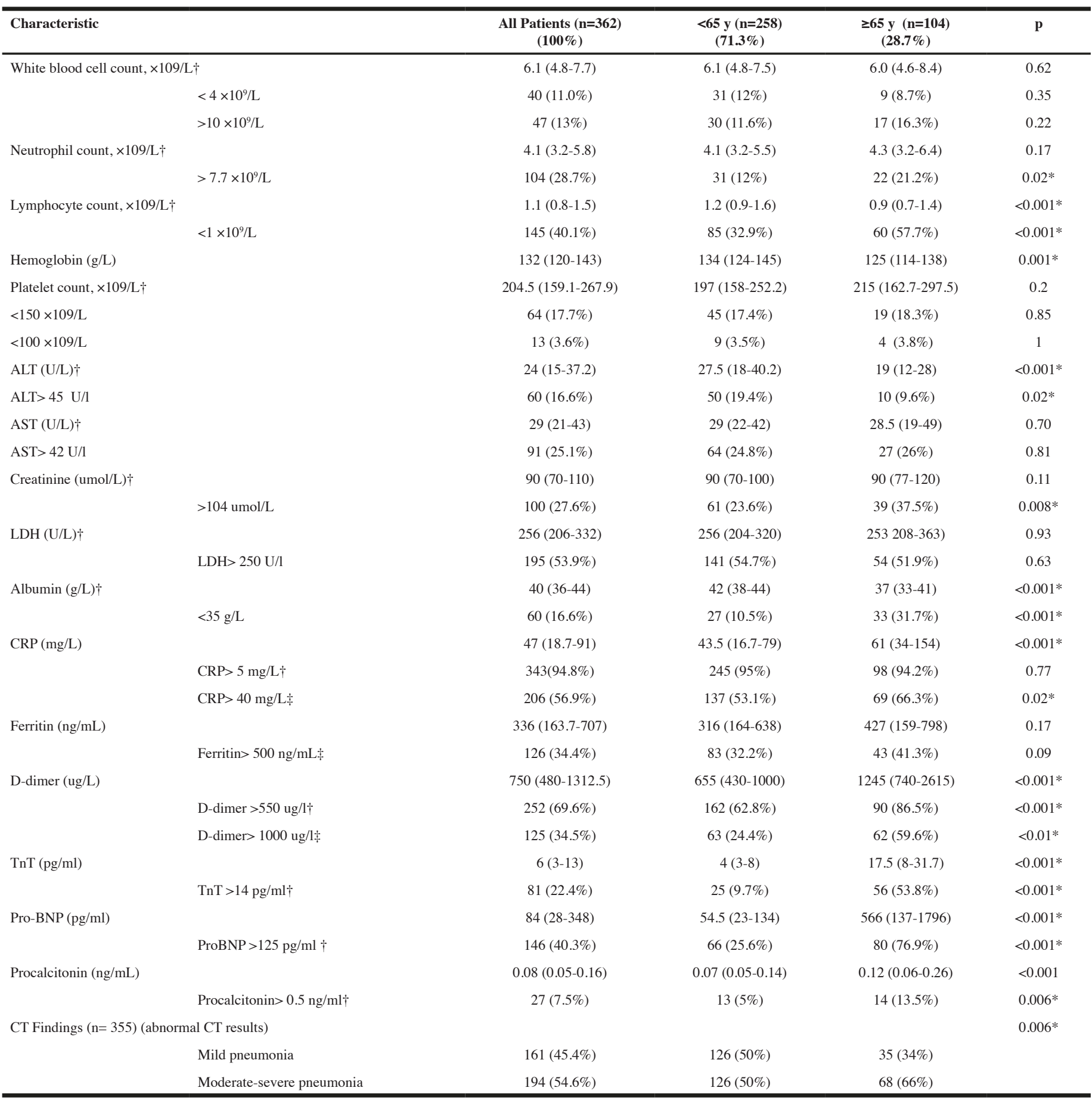

Data are given as median (interquartile range) for the continuous variables and as counts (\%) for the categorical variables; The cut-offs of the laboratory parameters were designated by $t$ the local laboratory thresholds and $\ddagger$ the suggestion of diagnostic and treatment guidelines for SARS-CoV-2 issued by the Turkish National Scientific Committee directed by the Turkish Ministry of Health (17); Admission laboratory studies were selected to be included here based on their relevance to the characterization of Covid-19 patients (5); CT: computerized tomography, ProBNP: N-terminal pro-brain natriuretic peptide, TnT: high sensitive troponin T; *statistically significant 


\section{OLDER ADULTS HOSPITALIZED WITH COVID-19}

Table 3

Outcome and follow-up characteristics of the hospitalized Covid-19 patients stratified by age

\begin{tabular}{|c|c|c|c|c|c|}
\hline Characteristic & & $\begin{array}{c}\text { All Patients }(n=362) \\
(100 \%)\end{array}$ & $\begin{array}{c}<65 \text { y }(n=258) \\
(71.3 \%)\end{array}$ & $\begin{array}{c}\geq 65 \text { y }(n=104) \\
(28.7 \%)\end{array}$ & $\mathbf{p}$ \\
\hline \multicolumn{6}{|l|}{ Outcome measures } \\
\hline & Admission to ICU & $53(14.6 \%)$ & $23(8.9 \%)$ & $30(28.8 \%)$ & $<0.001 *$ \\
\hline & Exitus & $35(9.7 \%)$ & $11(4.3 \%)$ & $24(23.1 \%)$ & $<0.001 *$ \\
\hline \multicolumn{6}{|l|}{ The time between (days) } \\
\hline & hospitalization to ICU admission & $2(0.5-6)$ & $2(0-8)$ & $2(1-5.2)$ & 1 \\
\hline & & $(\mathrm{n}=53)$ & $(n=23)$ & $(\mathrm{n}=30)$ & \\
\hline Length of hospital stay (days) $(n=362)$ & & $8.5(6-13)$ & $8(6-12)$ & $10(6-18)$ & $0.01 *$ \\
\hline \multirow[t]{2}{*}{ ICU duration (days) $(n=53)$} & & $26(10.5-46.5)$ & $15(7-35)$ & $36.5(14.2-48.2)$ & 0.06 \\
\hline & & $(\mathrm{n}=53)$ & $(n=23)$ & $(\mathrm{n}=30)$ & \\
\hline
\end{tabular}

Data are given as median (interquartile range) for the continuous variables and as counts (\%) for the categorical variables; ICU: intensive care unit; *statistically significant

median age for older adults ( $\geq 65$ years) was 73 years (IQR, 68-81 range, 65-90). More than half of the patients (58\%) had chronic comorbidity. The older patients had significantly more prevalent hypertension, diabetes mellitus, coronary heart disease, congestive heart failure, malignancy, a higher number of accompanying comorbidities and less prevalent smoking history ( $\mathrm{p}<0.01$ for all). Contact history was present in $37 \%$ of the population, older adults reporting significantly less contact history than the younger adults $(28.8 \%$ vs $40.3 \%, \mathrm{p}=0.04)$. Fatigue/myalgia (90.6\%), dry cough (81.2\%), and fever $(73.2 \%)$ were the three most common presenting symptoms in the study population with the prevalence in the older patients as $89.4 \%, 72.1 \%$, and $63.5 \%$, respectively. Cough and fever were significantly less prevalent in older adults compared to younger patients ( $\mathrm{p}=0.001$ and 0.008 , respectively). A higher percentage of the older adults complained of dyspnea at presentation, but the prevalence was not statistically different across the age groups ( $50 \%$ vs. $41.1 \%, \mathrm{p}=0.12)$. Median time from first symptom to hospitalization was 5 days (IQR, 3-7 days), and this was constant across the age groups. In the triage assessment, clinically severe pneumonia was present in $31.5 \%$ of the study population, and it was more common in older adults (49\% vs. $24.4 \%)(p<0.001)$. In line with this finding, respiration rate per minute was higher, room air $\mathrm{SaO} 2 \%$ was lower in the older adults with significantly higher rates of patients having respiration rate $\geq 30 / \mathrm{min}, \mathrm{SaO} 2<90 \%$ and requiring the use of accessory respiratory muscles among older adults $(\mathrm{p}<0.001$ for all). While triage body temperature and fever prevalence were not different across the older and younger patients, the prevalence of subfebrile temperature was also higher in the older adults $(20.2 \%$ vs. $10.9 \%, \mathrm{p}=0.002)$.

Laboratory and radiological findings at hospital admission

Table 2 shows the laboratory and radiological findings at hospital admission stratified by age. In the overall study population, lymphopenia was a prominent feature of the infection, $40.1 \%$ had lymphocyte $<1 \times 10^{9} / \mathrm{L}$, neutrophilia was present in $28.7 \%$, leukopenia and leukocytosis were each present in $10-15 \%$, and platelet $<100 \times 10^{9}$ in only $3.6 \%$. CRP was $>40 \mathrm{mg} / \mathrm{L}$ in more than half of the patients $(56.9 \%)$, D-dimer was higher than $1000 \mathrm{ug} / \mathrm{L}$ and ferritin was higher than $500 \mathrm{ng} / \mathrm{mL}$ in about $1 / 3$ patients $(34.5 \%$ and $34.4 \%$, respectively ), pro-BNP was elevated in $40.3 \%$, TnT was elevated in about $1 / 5$ patients $(22.4 \%)$, and procalcitonin was elevated in $7.5 \%$. LDH was increased in half of the patients $(53.9 \%)$, creatinine was increased in $27.6 \%$, ALT and AST was increased in $16.6 \%$ and $25.1 \%$. The laboratory parameters that were significantly different between the older and younger adults were as follows: the older patients had significantly higher CRP, D-dimer, TnT, pro-BNP, procalcitonin levels, higher prevalence of lymphopenia, neutrophilia, increased creatinine, and lower hemoglobin, ALT, albumin levels (Table 2). Chest CT was performed in almost all patients [358 (98.9\%) patients] except for four patients who were pregnant. 355 $(99.2 \%)$ patients had pneumonic infiltration. In more than half of the patients $(54.6 \%)$, there was moderate-severe pneumonia. Moderate-severe pneumonia was more prevalent among older patients $(66 \%$ vs $50 \%)(\mathrm{p}=0.006)$.

\section{Outcome and Follow-up Characteristics}

Outcome and follow-up characteristics are outlined in Table 3. ICU admission and death occurred in $14.6 \%(n=53)$ and $9.7 \%(n=35)$ of the patients, respectively. The adverse outcomes were significantly more prevalent in older adults compared to the younger patients (ICU admission, 28.8\% vs. $8.9 \%$; mortality, $23.1 \%$ vs. $4.3 \%$, p $<0.001$ for all). Time from hospitalization to ICU was similar in older and younger patients (median, 2 days), but the length of hospital stay was significantly longer in the older adults (median 10 vs. 8 days, $\mathrm{p}=0.01)$. In the patients that admitted to ICU, length of ICU stay was also higher in the older adults (36.5 days vs. 15 days). This difference was not statistically different, probably due to the lower number of patients admitted to ICU, but close to the significance level $(\mathrm{p}=0.06)$. 


\section{THE JOURNAL OF NUTRITION, HEALTH \& AGING}

\section{Factors associated with death in older adults}

Among 104 hospitalized older adults, 24 (23.1\%) died in the hospital follow-up. In the univariate analyses of the factors on admission, the factors associated with mortality were the absence of cough and presence of dyspnea by history; the presence of clinically severe pneumonia and its components (dyspnea, higher respiration rate, lower room air peripheral $\mathrm{SaO} 2$, higher pulse rate) on physical examination, and by laboratory examination; the presence of CRP $>40 \mathrm{mg} / \mathrm{L}$, higher D-dimer, TnT, pro-BNP, LDH, ALT, and lower albumin levels according to the laboratory thresholds, serum creatinine level and presence of moderate-severe pneumonia in CT $(\mathrm{p}<0.05$ for all). None of the comorbidities was specifically associated with mortality nor the number of comorbidities. After checking for multicollinearity, the presence of dyspnea and components/ indicators of the clinically severe pneumonia was not included in the regression. The covariates that were included in regression analyses were the presence of cough, clinically severe pneumonia on admission, presence of CRP $>40 \mathrm{mg} / \mathrm{L}$, higher D-dimer, TnT, pro-BNP, LDH, ALT, and lower albumin levels, serum creatinine and presence of moderate-severe pneumonia on CT. We ran Cox-regression analyses at first, but the model was not significant $(p=0.08)$. Therefore, we performed a logistic regression analysis. The significance of the logistic regression was acceptable $(\mathrm{R} 2=0.961$ (Hosmer and Lemeshow test), Model x2 (8)=2.5; p $<0.001)$. Among the triage evaluation parameters, the only factor associated with higher mortality was the presence of clinically severe pneumonia on admission (Odds Ratio $=12.3$, 95\% confidence interval $=2.7-55.5, \mathrm{p}=0.001)$.

Table 4

Logistic regression analyses for factors associated with death in older adults (Backward LR method)

\begin{tabular}{lccc}
\hline Characteristic & $\begin{array}{c}\text { Odds } \\
\text { Ratio }\end{array}$ & $\begin{array}{c}\mathbf{9 5 \%} \text { Confidence } \\
\text { Interval }\end{array}$ & p \\
\hline Clinically severe pneumonia on admission & 12.3 & $(2.7-55.5)$ & $0.001 *$ \\
Increased ALT (>45 U/L) & 2.3 & $(0.5-10.7)$ & 0.27 \\
Increased D-dimer (>550 ug/L) & 0 & 0 & 0.99 \\
Serum creatinine (mg/dL) & 1.7 & $0.97-3.0$ & 0.06 \\
\hline
\end{tabular}

Dependent variable: death; Independent variables: presence of cough, clinically severe pneumonia on admission, presence of CRP> $40 \mathrm{mg} / \mathrm{L}$, higher d-dimer, high sensitive troponin $\mathrm{T}, \mathrm{N}$-terminal pro-brain natriuretic peptide, $\mathrm{LDH}, \mathrm{ALT}$, and lower albumin levels by laboratory thresholds, serum creatinine and presence of moderate-severe pneumonia on computerized tomography; $(\mathrm{R} 2=0.961$ (Hosmer and Lemeshov test), Model x2 (8) $=2.5 ; \mathrm{p}<0.001) ; *$ statistically significant difference

\section{Discussion}

From the beginning of the pandemic, older adults have been more seriously affected by the outcomes of Covid-19. As an example, death over 60 years old accounted for $81 \%$ of the total deaths in China (6). In Turkey, cases $\geq 65$ years constituted $11 \%$ of the confirmed infections. The overall death rate was $2.6 \%$, but $70 \%$ of the overall deaths were in people aged 65 years or older (14). Despite the vulnerability of the older population, specific information about the clinical characteristics of the disease in older adults is limited in the literature (6-12). To our knowledge, only seven studies were focusing on older adults, all reported from China. The older patient numbers ranged between 18 and 60 in three studies (10-12), 105-136 in two of them $(8,9)$, and 204-339 patients in the two more comprehensive studies $(6,7)$. All the studies that included $>100$ older adults set the cut-off for older adults as 60 years. In this study, we included 104 older patients $\geq 65$ years, which accounted for $28.7 \%$ of hospitalized patients in the study period. Therefore, this study aimed to be one of the studies, including a high number of older adults among studies focusing on older adults and thereby add data to increase our knowledge on clinical characteristics of Covid-19 in this group.

As reported in the other studies, the older adults had more prevalent associated comorbidities compared to the younger patients, hypertension (62.5\%), diabetes mellitus (32.7\%), coronary heart disease $(25 \%)$ being the top three comorbidities. Of note, hypertension and diabetes were more common in this study than in all of the Chinese studies of older adults with Covid-19 which were between $27.8 \%-48.4 \%$ and $7.9-25.7 \%$, respectively, This may be due to ethnical differences in the prevalences of these chronic diseases probably reflecting the lower prevalence of hypertension and diabetes among Chinese people compared to the nonhispanic whites $(19,20)$. Older adults had less prevalent contact history, and this is probably due to the early isolation of older adults starting from the beginning of the pandemic. This reflects that older adults are more prone to develop the infection with lower microorganism load, probably indicating that asymptomatic infectious patients were an important source of Covid-19 infection in older adults.

The most common presenting symptoms were similar across the age groups as fatigue/myalgia, dry cough, and fever being $89.4 \%, 72.1 \%$, and $63.5 \%$ in the older adults. Dyspnea was also very prevalent, affecting half of the older adults. Dry cough and fever are, in general, the two most common symptoms of Covid-19 in older adults, reported between 33.3-69.1\% and 40.4-94.5\% in the reports in China. In our structured history, we asked for fatigue and/or myalgia together, and this symptom was the most common presenting symptom differing from the other reports. Cough and fever were less prevalent, and subfebrile fever at the triage was more prevalent in older adults, probably reflecting the decreased host response-ability in the elderly. However, in a study including 136 older adults $\geq 60$ years of age, fever was more prevalent among older adults (8). Thus, this is not a consistent finding and needs to be addressed in future studies. Importantly, clinically severe pneumonia which was identified by the presence of triage respiration rate $\geq 30 / \mathrm{min}$ or resting room air peripheral oxygen saturation $<90 \%$ or dyspnea (detected by use of accessory respiration muscles at rest) was present in about half of the older adults $(49 \%)$. It was significantly more prevalent than in the younger 


\section{OLDER ADULTS HOSPITALIZED WITH COVID-19}

patients $(24.4 \%)$, while the time from the first symptom to hospitalization was not different. Admission of older adults with severe clinical disease compared to younger adults is also a constant finding across the studies $(8,10-12)$. As the time from the first symptom to hospitalization was not different between the older and younger adults, these results indicate that the infection probably displays a more rapid course in older adults. Another factor may be, as we found less common fever and cough among older adults, the older adults might have had progression of the disease before being symptomatic classically.

The common laboratory findings of the hospitalized Covid-19 patients at the triage were significantly elevated CRP (>40 mg/L), high pro-BNP and LDH, lymphopenia, high ferritin and D-dimer levels, neutrophilia, high creatinine, AST, TnT levels and presence of moderate-severe pneumonic infiltration in descending order (Table 2). The significantly different laboratory findings between the older and younger patients were also indicators of more severe involvement with more prevalent abnormalities in the laboratory evaluation i.e. CRP, D-dimer, TnT, pro-BNP, procalcitonin, lymphopenia, neutrophilia, creatinine, hemoglobin, ALT, albumin and more prevalent moderate-severe pneumonia (66\% vs. $50 \%)$ on CT. Our findings are in accordance with the other studies $(8,11,12)$

The mortality rate was $9.7 \%$ in the total study population, which has a median age of 56 years. The mortality rate was much higher in older patients compared with the younger counterparts ( $23.1 \%$ vs. $4.3 \%, \mathrm{p}<0.001)$. The ICU admission, as an indicator of severe disease, was $14.6 \%$ in the general study population, and this was also much more prevalent in the older adults $(28.8 \%$ vs. $8.9 \%, \mathrm{p}<0.001)$ occurring at a median of 2 days after hospitalization. These figures also reflect the rapid course and more prevalent adverse outcomes of the Covid-19 in older adults, which is in line with the previous recent studies $(8,12)$. The fatality rate among older adults has been reported higher as $37.3 \%$ in which the authors noted that most of the patients had to be isolated at home without medical support due to lack of in-patient hospital beds (7). This point is, therefore, important suggesting that again, earlier supportive measures may improve prognosis in older adults.

In this study, we analyzed the triage factors that were related to the in-hospital mortality of older adults. The older adults that died had many significant abnormal laboratory findings reflecting more extensive involvement of the disease and associated with poor prognosis suggested in the published Covid-19 literature (e.g., higher inflammatory markers, a variety of biochemical abnormalities, and presence of moderate-severe pneumonia in CT). This data can help physicians in stratifying patients with higher and lower risk according to the triage assessment. Of note, in the multivariate analysis, the only factor at triage assessment related to mortality was the presence of clinically severe pneumonia on admission. This is valuable as it supports the general medical principle that the clinical evaluation of the patient is more important than the laboratory examinations in medical assessment. This result has also been reported indirectly, as the presence of dyspnea was an independent correlate of mortality in the studies that could have performed multivariate analysis for correlates of mortality in the older adults $(6,7,12)$. It seems that the more extensive pulmonary involvement on presentation reflected by the presence of dyspnea, tachypnea, and low oxygen saturation, is probably the main driver of the fatality in the course of this disease. Our findings, together with the others, support the suggestion that older adults should be evaluated carefully for early diagnosis of the disease, which may hopefully reduce mortality rates by the introduction of supportive respiratory measures earlier $(7,12)$. On the other hand, other studies noted some additional factors, with some differences between each other associated with higher mortality, e.g., older age, male gender, neutrophilia, lymphopenia, elevated troponin or D-dimer levels, underlying chronic diseases, cardiovascular diseases, chronic obstructive pulmonary disease, elevated creatinine and procalcitonin levels $(6,7,12)$.

This study has several limitations. It is a single-center retrospective study with a limited patient number, and we considered only the triage factors but not included the progress data and treatment modalities. However, it was the aim of this study to describe the presenting clinical characteristics of Covid-19 on admission and among them to identify the factors associated with higher mortality among older adults. Follow-up data that could enable clinicians to stratify patients as having lower or higher risk likely present, but this was beyond the scope of this study. While we did not include treatment details, as the treatments were managed by a constant team, a significant difference between the management of the patients was unlikely but could not be eliminated by the study design. An important strength of the study is, we introduced a structured approach to the triage patients, and therefore, there is no missing data except for the data on less frequent potential presenting symptoms of the infection (loss of smell/ taste and gastrointestinal symptoms). Also, in this study, we had complete data for the in-hospital outcome for all patients.

In conclusion, this study shows that older Covid-19 patients that were hospitalized presented with more prevalent chronic comorbidities, less prevalent symptomatology (i.e., cough and fever) but more severe respiratory signs and laboratory abnormalities than the younger patients. Among the triage assessment factors, while there were several laboratory indicators related to mortality of the older adults, the clinical evaluation of pulmonary involvement came front to help clinicians to stratify the patients for mortality risk.

Ethical approval: The study was approved by the institutional review board (number: 2020/747).

Author Contributions: Concept and design: Alpay Medetalibeyoğlu, Gülistan Bahat, Mustafa Erelel, Mustafa Oral Oncül, Tufan Tükek. Acquisition, analysis, or interpretation of data: Naci Şenkal, Murat Köse, Yunus Çatma. Preparation and revisions to scientific content of manuscript: Naci Şenkal, Yunus Çatma; Critical revision of the manuscript: Gülistan Bahat, Murat Köse, Alpay Medetlibeyoğlu ; Stylistic/grammatical revisions of the manuscript and drafting: Emine Bilge Çaparali. 


\section{THE JOURNAL OF NUTRITION, HEALTH \& AGING}

Conflict of Interests: Alpay Medetalibeyoğlu declares that there is no conflict of interest. Naci Şenkal declares that there is no conflict of interest. Murat Köse declares that there is no conflict of interest. Yunus Çatma declares that there is no conflict of interest. Emine Bilge Çaparali declares that there is no conflict of interest. Mustafa Erelel declares that there is no conflict of interest. Mustafa Oral Öncül declares that there is no conflict of interest. Gülistan Bahat Öztürk declares that there is no conflict of interest. Tufan Tükek declares that there is no conflict of interest.

Acknowledgements: The authors would like to thank Dr. Merve Meryem Ören for her contributions to statistical analysis.

\section{References}

1. Huang C, Wang Y, Li X et al. Clinical features of patients infected with 2019 novel coronavirus in Wuhan, China. Lancet. 2020;395(10223):497-506. doi.org/10.1016/ S0140-6736(20)30183-5

2. Zheng J. SARS-CoV-2: an emerging coronavirus that causes a global threat. Int J Bio Sci 2020;16(10):1678-85. doi: 10.7150/ijbs.45053

3. WHO Coronavirus Disease (COVID-19) Dashboard. https://covid19.who.int/ accessed on July 2, 2020

4. Wu Z, McGoogan JM. Characteristics of and important lessons from the coronavirus disease 2019 (COVID-19) outbreak in China: summary of a report of 72314 cases from the Chinese center for disease control and prevention. JAMA 2020;323(13):1239-1242. doi:10.1001/jama.2020.2648

5. Richardson S, Hirsch JS, Narasimhan M, et al. Presenting characteristics, comorbidities, and outcomes among 5700 patients hospitalized with COVID-19 in the New York City Area. JAMA. 2020;323(20):2052-2059. doi:10.1001/jama.2020.6775

6. Wang $\mathrm{L}, \mathrm{He} \mathrm{W}, \mathrm{Yu} \mathrm{X}$, et al. Coronavirus disease 2019 in elderly patients: characteristics and prognostic factors based on 4-week follow-up. J Infect. 2020;80(6):639-645. doi:10.1016/j.jinf.2020.03.019

7. Li P, Chen L, Liu Z, et al. Clinical features and short-term outcomes of elderly patient with COVID-19. Int J Infect Dis 2020;97:245-250. doi:10.1016/j.ijid.2020.05.107

8. Lian J, Jin X, Hao S, et al. Analysis of epidemiological and clinical features in older patients with corona virus disease 2019 (COVID-19) out of Wuhan Clin Infect Dis. 2020;ciaa242. doi:10.1093/cid/ciaa242
9. Guo T, Shen Q, Guo W et al. Clinical characteristics of elderly patients with COVID19 in Hunan province, China: a multicenter, retrospective study. Gerontology. 2020:1 9. doi: $10.1159 / 000508734$.

10. Niu S, Tian S, Lou J, et al. Clinical characteristics of older patients infected with COVID-19: a descriptive study. Arch Gerontol Geriatr. 2020;89:104058. doi:10.1016/j.archger.2020.104058

11. Liu K, Chen Y, Lin R, Han K. Clinical features of COVID-19 in elderly patients: a comparison with young and middle-aged patients. J Infect. 2020;80(6):e14-e18. doi:10.1016/j.jinf.2020.03.005

12. Chen T, Dai Z, Mo P et al. Clinical characteristics and outcomes of older patients with coronavirus disease 2019 (COVID-19) in Wuhan, China (2019): a single-centered, retrospective study. J Gerontol A Biol Sci Med Sci. 2020;glaa089. doi:10.1093/gerona/ glaa089

13. http://tuik.gov.tr/UstMenu.do?metod=temelist; accesed on July 2, 2020 .

14. COVID-19 Situation Report Turkey https://dosyamerkez.saglik.gov.tr/ Eklenti/37743,covid-19-situation-report-v4pdf.pdf?0 accessed on July 2, 2020.

15. World Health Organization. Laboratory testing for 2019 novel coronavirus (2019-nCoV) in suspected human cases. Interim guidance. https://www.who.int/ publications/i/item/10665-331501 accessed on July 2, 2020.

16. Ooi GC, Khong PL, Müller NL, et al. Severe acute respiratory syndrome: temporal lung changes at thin-section CT in 30 patients. Radiology. 2004;230(3):836-844 doi:10.1148/radiol.2303030853

17. Guides of COVID-19 https://covid19bilgi.saglik.gov.tr/depo/rehberler/COVID19_Rehberi.pdf accessed June 1, 2020.

18. Vittinghoff $\mathrm{E}, \mathrm{McCulloch} \mathrm{CE}$. Relaxing the rule of ten events per variable in logistic and cox regression. Am J Epidemiol. 2007;165(6):710-718. doi:10.1093/aje/kwk052

19. Zhao B, Jose PO, Pu J, et al. Racial/ethnic differences in hypertension prevalence, treatment, and control for outpatients in northern California 2010-2012. Am J Hypertens. 2015;28(5):631-639. doi:10.1093/ajh/hpu 189

20. Spanakis EK, Golden SH. Race/ethnic difference in diabetes and diabetic complications. Curr Diab Rep. 2013;13(6):814-823. doi:10.1007/s11892-013-0421-9 